\title{
Assessment of spring cranioplasty biomechanics in sagittal craniosynostosis patients
}

\author{
Alessandro Borghi, PhD, 1,2 Silvia Schievano, PhD,,2 Naiara Rodriguez Florez, PhD,,2 \\ Roisin McNicholas, ${ }^{2}$ Will Rodgers, MRCS, ${ }^{1,2}$ Allan Ponniah, FRCS(Plast), ${ }^{2}$ \\ Greg James, PhD, FRCS, ${ }^{2}$ Richard Hayward, FRCS(Eng), ${ }^{2}$ David Dunaway, FRCS(Plast), ${ }^{1,2}$ and \\ N. u. Owase Jeelani, FRCS(NeuroSurg) ${ }^{1,2}$ \\ IUCL Great Ormond Street Institute of Child Health; and ${ }^{2}$ Great Ormond Street Hospital for Children, London, United Kingdom
}

OBJECTIVE Scaphocephaly secondary to sagittal craniosynostosis has been treated in recent years with spring-assisted cranioplasty, an innovative approach that leverages the use of metallic spring distractors to reshape the patient skull. In this study, a population of patients who had undergone spring cranioplasty for the correction of scaphocephaly at the Great Ormond Street Hospital for Children was retrospectively analyzed to systematically assess spring biomechanical performance and kinematics in relation to spring model, patient age, and outcomes over time.

METHODS Data from 60 patients (49 males, mean age at surgery $5.2 \pm 0.9$ months) who had received 2 springs for the treatment of isolated sagittal craniosynostosis were analyzed. The opening distance of the springs at the time of insertion and removal was retrieved from the surgical notes and, during the implantation period, from planar radiographs obtained at 1 day postoperatively and at the 3-week follow-up. The force exerted by the spring to the patient skull at each time point was derived after mechanical testing of each spring model-3 devices with the same geometry but different wire thicknesses. Changes in the cephalic index between preoperatively and the 3-week follow-up were recorded.

RESULTS Stiffer springs were implanted in older patients $(p<0.05)$ to achieve the same opening on-table as in younger patients, but this entailed significantly different-higher-forces exerted on the skull when combinations of stiffer springs were used $(p<0.001)$. After initial force differences between spring models, however, the devices all plateaued. Indeed, regardless of patient age or spring model, after 10 days from insertion, all the devices were open.

CONCLUSIONS Results in this study provide biomechanical insights into spring-assisted cranioplasty and could help to improve spring design and follow-up strategy in the future.

https://thejns.org/doi/abs/10.3171/2017.1.PEDS16475

KEY WORDS craniosynostosis; spring cranioplasty; mathematical model; $x$-ray; craniofacial

$\mathrm{S}$ PRING-assisted cranioplasty, first introduced by Lauritzen et al. in 1998, is a surgical technique for the treatment of patients with sagittal craniosynostosisrelated scaphocephaly. ${ }^{11}$ Possible advantages of the technique, as compared with more traditional formal cranial vault remodeling, include lower blood loss, transfusion requirements, and overall hospital costs, ${ }^{12}$ while appearing comparable in efficacy and safety. ${ }^{13}$ The biomechanical effect of spring cranioplasty has been investigated in animal models in vivo and in vitro, showing that the procedure alters the natural growth vector of the skull, ${ }^{6}$ produces su- pra-physiological strains in sutural and parasutural tissue, ${ }^{4}$ causes bone thickening, ${ }^{3}$ and promotes overall global cranial expansion by changing cephalometric angles. ${ }^{8}$ In particular, animal models have revealed that springs achieve $80 \%$ expansion after 2 weeks of insertion ${ }^{7}$ and that $28 \%$ residual force is found at the time of removal. ${ }^{3}$

One of the anxieties about spring-assisted cranioplasty for scaphocephaly has been the lack of available standardized distractors and surgical approaches, which limits understanding of the biomechanical interaction between the device and the skull and raises concerns about the pre-

ABBREVIATIONS $\triangle \mathrm{Cl}$ = change in cephalic index; FU1 = first follow-up 1 day after surgery; FU2 = second follow-up approximately 3 weeks after surgery; GOSH = Great Ormond Street Hospital for Children; OP = spring opening distance.

ACCOMPANYING EDITORIAL See pp 397-399. DOI: 10.3171/2017.3.PEDS1725.

SUBMITTED August 18, 2016. ACCEPTED January 16, 2017.

INCLUDE WHEN CITING Published online August 25, 2017; DOI: 10.3171/2017.1.PEDS16475. 
dictability of surgical results. Spring opening forces in patients have been assessed on the operating table; ${ }^{2,13,15,17,19}$ however, no information is currently available on spring biomechanics after implantation, during follow-up, and at removal in patients.

Spring-assisted cranioplasty was introduced at the Great Ormond Street Hospital for Children (GOSH), London, in 2010 for the correction of scaphocephaly in sagittal synostosis, using preformed spring devices and a standardized surgical approach. The GOSH devices are prefabricated torsional springs (The Active Spring Company) made of stainless steel wire with a central loop and an initial opening of $60 \mathrm{~mm}$ between the tips at rest, before implantation (Fig. 1). Three standardized models with the same geometry but different wire thicknesses for varying stiffness are currently in use: models S10, S12, and S14 with 1.0-, 1.2-, and 1.4-mm thicknesses, respectively. Design standardization ensures reproducibility of the force/ opening behavior for each spring model.

The aim of this study was to fully characterize, through a retrospective analysis of available clinical data, the GOSH springs and their biomechanical interaction with the skull in patients treated for sagittal craniosynostosis. In vivo spring mechanical performance and distraction kinematics during the time of implantation were derived from on-table measurements at insertion and removal and from radiographic images at follow-up.

\section{Methods}

\section{Surgical Procedure}

The procedure for scaphocephaly correction involves an $8-\mathrm{cm}$ linear biparietal skin incision with subgaleal dissection to the anterior and posterior fontanelles, followed by a $15-\mathrm{mm}$ square craniectomy in the center of the fused sagittal suture to permit visualization and complete dural dissection up to, and slightly past, the coronal sutures anteriorly and the lambdoid sutures posteriorly. Two parasagittal osteotomies are fashioned to "free" the fused suture from the parietal bones bilaterally. Typically, 2 springs are inserted in the parasagittal cuts, 1 anteriorly and 1 posteriorly; the spring foot plates are positioned in small grooves created on the osteotomy lines and transfer the force exerted by the bending of the spring oblique arms onto the osteotomy edges. The grooves are typically made at the corners of the original craniectomy, but can be adjusted anteriorly or posteriorly for cases with asymmetric scaphocephaly. Spring strength selection is based on clinical assessment of the calvarial bone quality and surgeon experience: the spring is replaced with a stiffer one until satisfactory on-table opening is achieved $-3 \mathrm{~cm}$ from edge to edge of the parasagittal osteotomy. Generally, the surgeon starts with the model S10 for younger patients (age 4 months) and S12 for older patients (age 5 months and above). The devices are left in situ for approximately 3 months and then surgically removed. Monitoring during these months is provided by planar radiographic images (lateral and submental-occipital views) obtained at the first follow-up (FU1) 1 day after surgery and at the second follow-up (FU2) approximately 3 weeks after surgery.
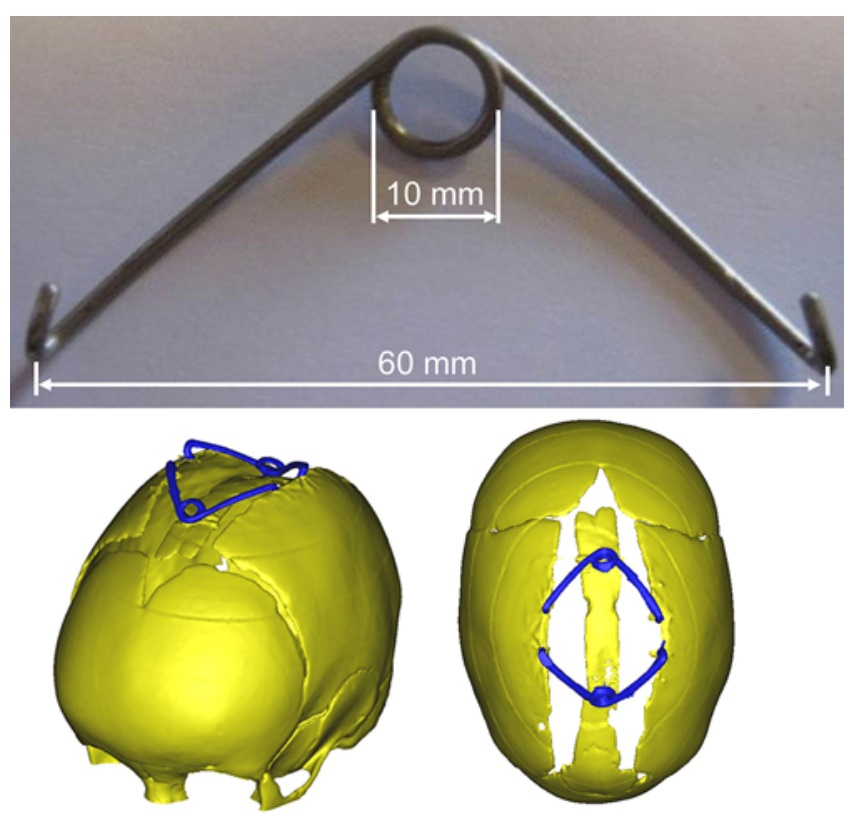

FIG. 1. A cranioplasty spring model (upper) used at GOSH. Sample 3D reconstruction of a patient skull after spring insertion (lower). Figure is available in color online only.

\section{Spring Mechanics}

The GOSH springs follow Hookean behavior: when compressed, they exert an outward force directly proportional to the amount of compression they have undergone. Therefore, crimping of the spring by the surgeon during the procedure to insert the spring in the grooves, which consists of decreasing the spring opening distance, translates into an outward force exerted by the distractor at the 2 ends (Fig. 2), which is directly proportional to the decrease in the spring opening distance (Eq. 1 below).

Two spring samples per model were mechanically tested to assess the distractor compressive behavior (basic force gauge, Mecmesin Ltd.; Fig. 2 left): each spring was crimped from the initial opening of $60 \mathrm{~mm}$ (resting conditions) to an opening of $20 \mathrm{~mm}$, similar to the crimped size at the time of implant, and back to $60 \mathrm{~mm}$. Foot plates (whose contribution to spring stiffness is negligible) were removed for ease of testing. Forces were recorded and values were averaged between the 2 samples for each opening stage. Force/opening curves were plotted during both the loading and unloading phase (Fig. 2 right). Linear trend lines were fitted to the latter since the springs progressively unload after implantation, and the spring working stiffness $(\mathrm{K})$ was calculated as the slope of the trend lines. The final opening postcrimping was estimated as the intersection of the trend line with the horizontal axis. Two further samples per spring were tested at a $30-\mathrm{mm}$ crimped distance instead of $20 \mathrm{~mm}$ to assess opening recovery and mechanical behavior in case of lower crimping.

\section{Patient Population and Image Analysis}

All patients referred to the GOSH supraregional Craniofacial Unit with a clinical diagnosis of scaphocephaly 

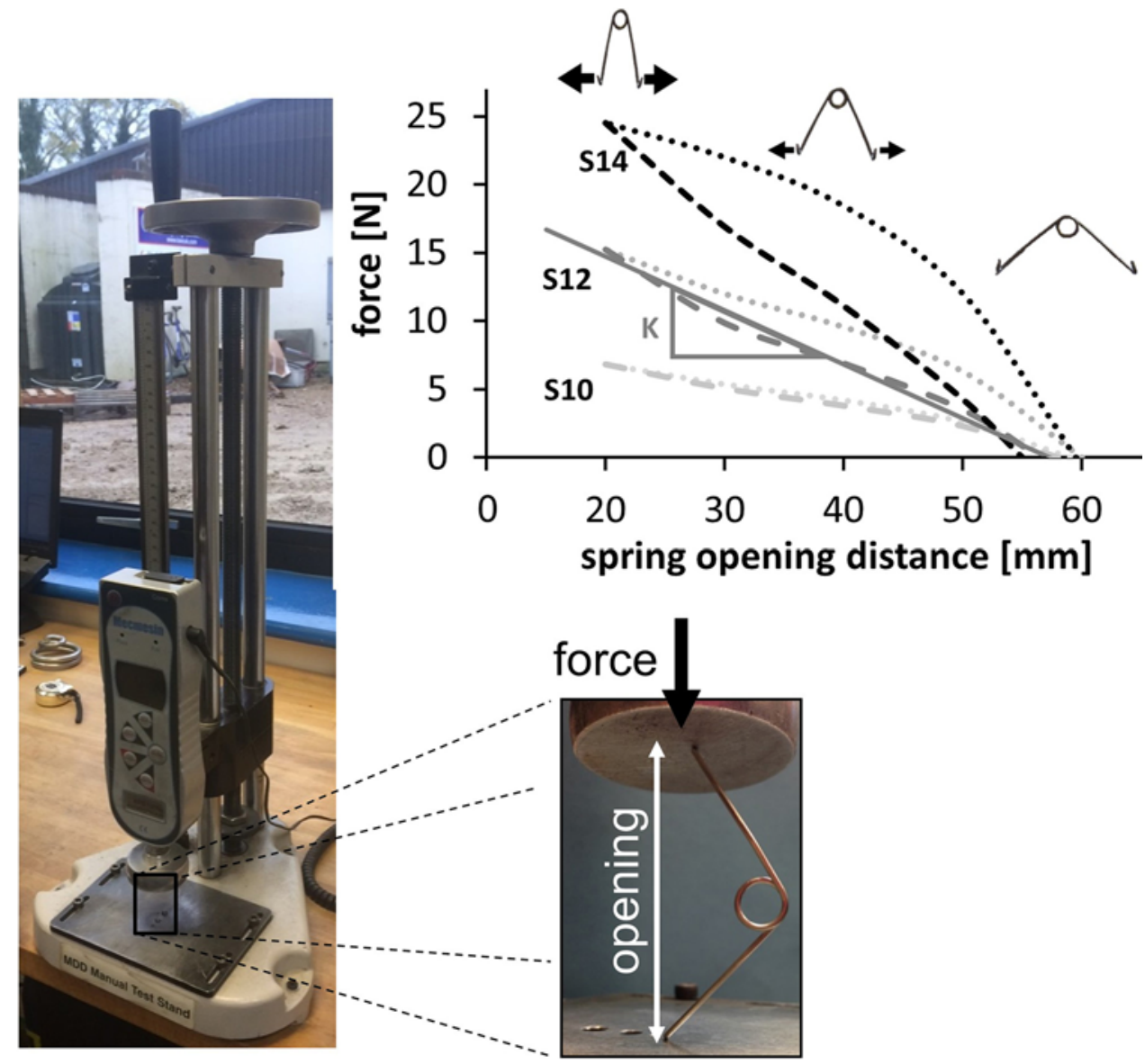

FIG. 2. Mechanical compression test (left) for the cranioplasty spring and graph (right) of the resulting force/opening curves for the different spring models at loading (dotted lines) and unloading (dashed lines). For the spring model S12, the linear trend fitting (solid line) used for the force calculation is visualized ( $\mathrm{K}$ is the spring stiffness). Figure is available in color online only.

are evaluated by a specialist craniofacial consultant, who confirms the diagnosis based on clinical examination and radiological imaging as necessary. In the presence of isolated sagittal synostosis with no functional issues in children 6 months of age or younger at the time of consultation, conservative management, modified pi procedure, ${ }^{9}$ and spring-assisted cranioplasty are discussed and offered as options to the family, who makes the final decision.

We retrospectively reviewed data from 93 consecutive cases of sagittal spring cranioplasty performed at GOSH between October 2010 and June 2013. Patients with multiple suture synostosis (5 patients), older than 7 months (17), who received more than 2 springs (7), who had complications at follow-up (spring reinsertion or expulsion, 2), and whose spring models could not be gleaned from the surgical notes (2) were excluded from this study, leaving a total of 60 patients.

Information on spring models, opening over time, and clinical outcome for these 60 patients was derived from the surgical notes and from radiographic images acquired preoperatively and postoperatively at the first and second follow-ups. Spring opening distance (OP), measured using a ruler at the time of spring insertion $\left(\mathrm{OP}_{\mathrm{I}}\right)$ and removal $\left(\mathrm{OP}_{\mathrm{R}}\right)$, was retrieved from the surgical notes; spring opening distance in situ over time was derived from skull planar radiographs in the submental-occipital view at the first follow-up $\left(\mathrm{OP}_{\mathrm{FU} 1}\right)$ and the second follow-up $\left(\mathrm{OP}_{\mathrm{FU}}\right)$. A previously validated geometric correction was adopted for the radiographic measurements to overcome geometric distortion due to skull shape and radiographic projection angle. ${ }^{10}$

The relationships between force and spring opening distance derived from the mechanical tests were used to estimate the force (F) exerted on the skull at each measured opening, according to the following equation (Eq. 1): $\mathrm{F}(\mathrm{t})=\mathrm{K}\left[\mathrm{OP}{ }_{\infty}-\mathrm{OP}(\mathrm{t})\right]$, where $\mathrm{K}$ and $\mathrm{OP}_{\infty}$ are the stiffness and opening values retrieved from the mechanical test of each spring model, respectively, and $\mathrm{OP}(\mathrm{t})$ is the spring opening distance at the different time points (insertion, first follow-up, second follow-up, and removal). A statistical multiple imputation method (implemented in SPSS 23, IBM Corp.) for a total of 15 iterations was used to account for missing data.

The effects of the spring model and age distribution on the opening outcomes were first analyzed. In addition, given that 2 different spring models can be used in the same child, to assess the effect of the combined spring system on each patient, openings of the anterior and poste- 
rior springs were averaged; thus, we identified 5 subgroups according to the spring combination: S10-S10, S10-S12, S12-S12, S12-S14, and S14-S14. Similarly, the combined force from the 2 springs acting on each patient skull was calculated as the sum of the forces of the anterior and posterior springs.

Changes in the cephalic index $(\Delta \mathrm{CI})$, derived from preoperative radiographs $\left(\mathrm{CI}_{\mathrm{PRE}}\right)$ and from radiographs obtained at the time of the second follow-up $\left(\mathrm{CI}_{\mathrm{FU} 2}\right)$, were calculated as a measure of outcome for the overall procedure: $\Delta \mathrm{CI}=\mathrm{CI}_{\mathrm{FU} 2}-\mathrm{CI}_{\mathrm{PRE}}$.

\section{Spring Kinematics}

The spring kinematics were analyzed using the opening values at the 4 time points, from insertion to removal. The skull was considered viscoelastic, ${ }^{14}$ and the spring opening distance was assumed to follow an exponential model governed by the time constant $\tau$ (Eq. 2):

$$
O P(t)=O P_{I O}+\left(O P_{R O}-O P_{I O}\right) \cdot\left(1-e^{-\frac{t}{\tau}}\right) .
$$

In an exponential rise, $\tau$ is the time at which the analyzed quantity reaches $(1-1 / e)=67 \%$ of its maximum value. In this case, $\tau$ is the time it takes (from insertion) for each implanted spring to reach $67 \%$ of its maximum opening.

Model fitting was achieved using the nonlinear least squares method, implemented in MATLAB (MathWorks). The value of the time constant $\tau$ was calculated for each patient, and the normal probability distribution of the data was tested after outlier removal (Kolmogorov-Smirnov test) to calculate the average and standard deviation of $\tau$ in the population ( $\mu_{\tau}$ and $\sigma_{\tau}$, respectively). The following 2 properties of exponential functions and normal distributions were combined to assess the timing of spring kinematics: 1) an exponential rise reaches its plateau after a time $\mathrm{T}=5 \tau$; and 2 ) in a normal distribution having a mean value $\mu$ and standard deviation $\sigma$, according to Chebyshev's inequality, $97.8 \%$ of the population lies within the interval $[-\infty, \mu+2 \sigma]$. Therefore, at a time $T=5\left(\mu_{\tau}+2 \sigma_{\tau}\right)$, $97.8 \%$ of the springs reach the opening plateau.

The value of each spring opening at time $\mathrm{T}\left(\mathrm{OP}_{\mathrm{T}}\right)$ was extrapolated from the patient exponential fitted curve and compared with the opening measured at removal $\left(\mathrm{OP}_{\mathrm{R}}\right)$ to assess and validate the analytical method used to describe the spring behavior.

\section{Statistical Analysis}

The Kolmogorov-Smirnov test was employed to assess group normality. The Wilcoxon rank test or t-test was used to analyze the difference in patient age, as well as spring opening distance and exerted force, between the spring model groups. The Pearson correlation was applied to investigate the relationship between age and combined force/opening at insertion, first follow-up, second followup, and removal, and $\Delta \mathrm{CI}$, whereas a paired t-test was used to perform statistical comparisons between paired results at each time point (insertion vs FU1, insertion vs FU2, insertion vs removal, FU1 vs FU2, and so forth). Finally, a Bland-Altman plot was adopted to highlight the differ-
TABLE 1. Stiffness and final opening for the $3 \mathrm{GOSH}$ spring models

\begin{tabular}{ccc}
\hline Spring Model & $\begin{array}{c}\text { Unloading Stiffness, } \\
\mathrm{K}(\mathrm{N} / \mathrm{mm})\end{array}$ & $\begin{array}{c}\text { Final Opening, } \\
\mathrm{OP}_{\infty}(\mathrm{mm})\end{array}$ \\
\hline $\mathrm{S} 10$ & 0.17 & 60.7 \\
\hline $\mathrm{S} 12$ & 0.39 & 57.3 \\
\hline $\mathrm{S} 14$ & 0.68 & 55.6 \\
\hline
\end{tabular}

ences between analytical fitting and real measurements for the spring opening distance at removal. A p $<0.05$ was considered statistically significant.

\section{Results \\ Spring Mechanics}

The spring showed nonlinear behavior at both loading and unloading (Fig. 2). The linearized unloading stiffness $(\mathrm{K})$ and final opening postcrimping $\left(\mathrm{OP}_{\infty}\right)$ for the different spring models are reported in Table 1 . In addition, the spring mechanical tests highlighted the presence of nonrecoverable deformations due to the yielding of stainless steel when the wire was crimped to smaller openings. Indeed, if crimped to $30 \mathrm{~mm}$ only instead of $20 \mathrm{~mm}$ (Fig. 3), model S14 presented better recovery of the original opening at release but at the expense of $25 \%$ lower force when compressed. This behavior was less evident for model S10
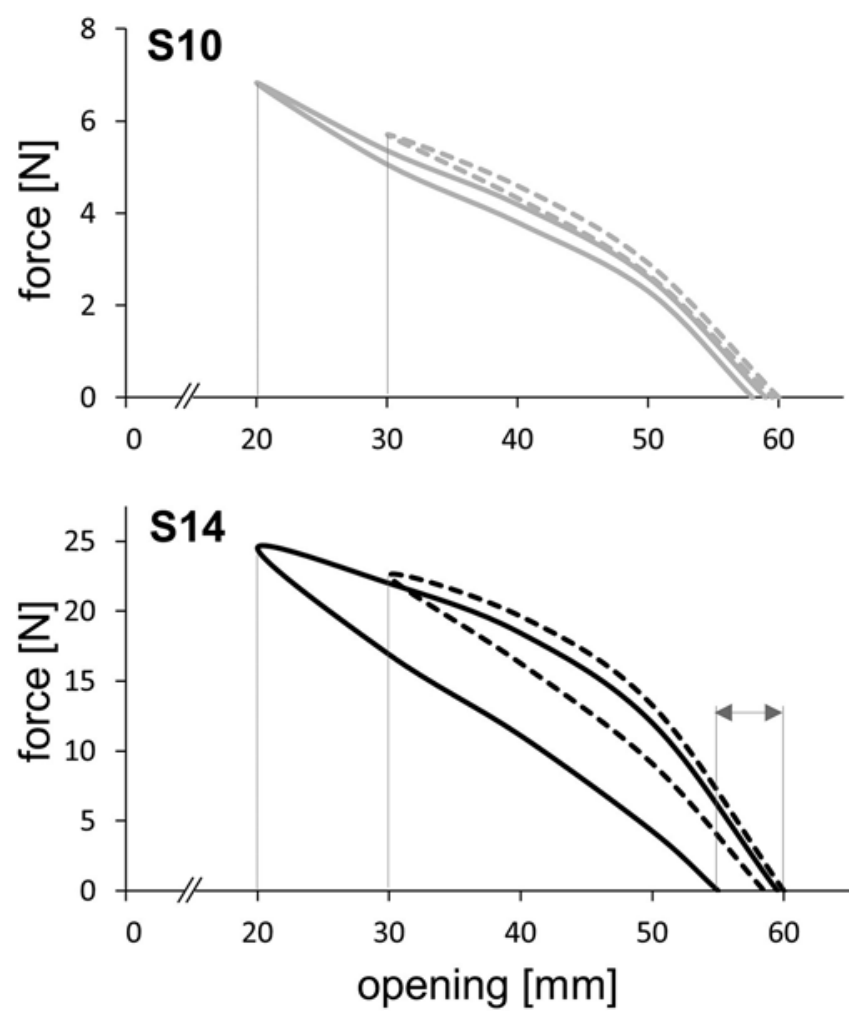

FIG. 3. Force/opening curve comparisons for models $S 10$ (upper) and $\mathrm{S} 14$ (lower), crimped to $20 \mathrm{~mm}$ (continuous lines) and $30 \mathrm{~mm}$ (dashed lines). Arrows indicate the permanent set (unrecoverable expansion) of the S14 when crimped to $20 \mathrm{~mm}$ before implantation. 

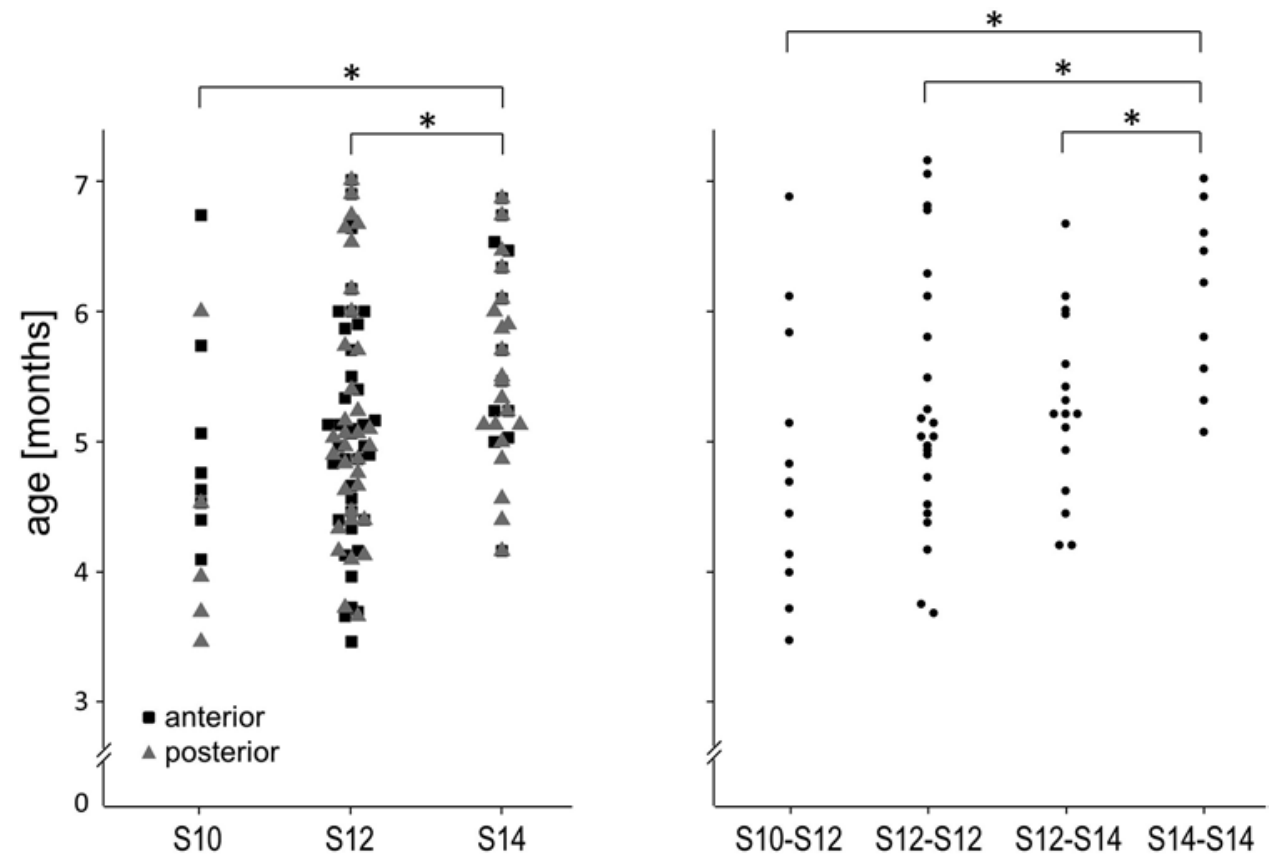

FIG. 4. Left: Age distribution for model S10, S12, and S14 springs positioned anteriorly (black squares) and posteriorly (gray triangles). Right: Age distribution for the combined spring system per patient. Asterisks indicate statistically significant differences.

because of the lower stresses that develop in the thinner wire, with only $11.2 \%$ difference in force and nearly full recovery of the opening when unloaded.

\section{Patient Population and Image Analysis}

Average patient age at the time of operation for the 60 selected patients was $5.2 \pm 0.9$ months (range 3.5-7.0 months) with a 5:1 male/female ratio. Springs remained implanted in the 60 patients for $110.9 \pm 53.6$ days (range 28-300 days) from insertion to removal. The time of the first follow-up was $1.05 \pm 0.22$ days (range 1-2 days; 60 patients), whereas the second follow-up happened after $22.6 \pm 9.0$ days (range 6-59 days; 57 patients with available radiographs). The analyzed population received a total of 120 springs: 13 model S10, 73 model S12, and 34 model S14. Patients receiving the S14 were significantly older (5.6 \pm 0.8 months) than those receiving the S10 (4.7 \pm 0.9 months, $\mathrm{p}=0.0036)$ and the S12 (5.1 $\pm 0.8, \mathrm{p}=0.0051$; Fig. 4 left).

Considering the spring combination per patient, only 1 child had the S10 implanted both anteriorly and posteriorly, 23 patients received two S12 springs, and 9 patients received two S14 springs. The remaining patients had a combination of 2 different spring models in the anterior and posterior positions, with no obvious trend for the position of the stiffer spring: 11 children received one S10 and one S12, whereas 16 patients had one S12 and one S14 (Fig. 4 right). Patients of the S14-S14 group were older (6.0 \pm 0.7 months) than those in the other groups (S10-S12: $4.8 \pm 1.0$ months, $p=0.007$; S12-12: $5.2 \pm 1.0$ months, $p=$ 0.034 ; and S12-14, $5.2 \pm 0.7$ months, $\mathrm{p}=0.009$ ), confirming that older patients received stiffer spring systems.

The combined opening and force plots over time showed the progressive spring opening from insertion to removal, while spring forces decayed (Fig. 5 and Table 2), with a statistical difference in opening and force between each consecutive time point $(\mathrm{p}<0.001$ for each time point comparison, except for the second follow-up vs removal when $p=0.0247$ for opening and $p=0.0296$ for force).

In terms of spring opening distance, there was no statistically significant difference between the 3 spring models at any point in time except at removal when the S10 springs opened less than the S12 $(p=0.0231)$. This was confirmed by our analysis of the combined opening as overall effect per patient, where only the S12-S12 combination showed lower opening than S12-S14 at removal ( $p$ $=0.0332$ ).

However, when considering forces, the S10 exerted lower force than the S12 and S14 at both insertion (p < 0.001 in both cases) and the first follow-up ( $\mathrm{p}=0.02$ and 0.0019 , respectively), and the S12 exerted lower force than the S14 at insertion $(\mathrm{p}<0.001)$ and the first follow-up ( $=0.001)$. No statistical difference in force was found between the models at the second follow-up and removal. When studying the combined forces, a statistically significant difference was found between each subgroup at the time of insertion. The S10-S12 subgroup exerted lower combined forces than S12-S14 ( $p=0.020)$ and S14-S14 (p $=0.013)$ at the first follow-up, whereas S12-S12 exerted lower combined forces than S14-S14 $(p=0.014)$ at the same time point.

The relationship between combined opening and age at insertion (Fig. 6) highlighted a moderate negative correlation at insertion $(\mathrm{r}=-0.39, \mathrm{p}=0.002)$, the first follow-up $(\mathrm{r}=-0.27, \mathrm{p}=0.034)$, and the second follow-up $(\mathrm{r}=-0.32$, $\mathrm{p}=0.011)$. No correlation was found at removal. A mod- 

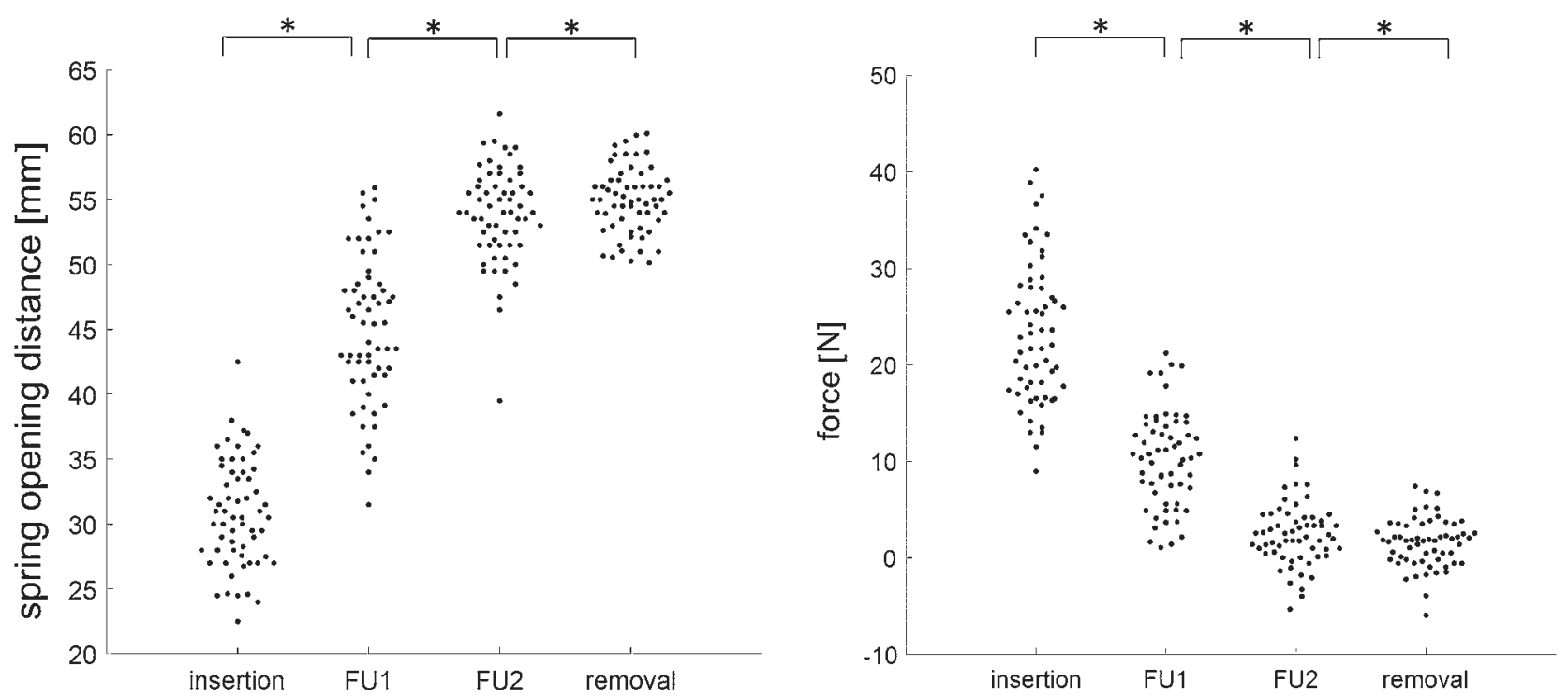

FIG. 5. Combined spring opening distance (left) and force (right) at insertion, FU1, FU2, and removal for each patient. Statistical difference was found between measurements at consecutive time points. Asterisks indicate statistically significant differences.

erate positive correlation was found between combined force and age at insertion $(r=0.48, p<0.001)$ and the first follow-up ( $\mathrm{r}=0.41, \mathrm{p}=0.001)$, with no correlation at the second follow-up and removal (Fig. 7).

An increase in the cephalic index from preoperatively to the second follow-up was negatively correlated with age $(\mathrm{r}=-0.38, \mathrm{p}=0.01$; from 45 patients who had both preoperative and second follow-up radiographs available; Fig. 8).

\section{Spring Kinematics}

The average value of the time constant $\tau$ retrieved from the model fitting (54 patients, after removing 5 outliers and 1 case with low goodness-of-fit) was $1.16 \pm 0.46$ days (range 0.46-2.76 days). Thus, the population time $\mathrm{T}$ was calculated as 10 days, implying that, after 10 days, $97.8 \%$ of the springs reached the opening plateau.

A comparison between spring opening distance at 10 days $\left(\mathrm{OP}_{\mathrm{T}}\right.$ evaluated by interpolation using the exponential model) and the value recorded on the operating table at the time of removal $\left(\mathrm{OP}_{\mathrm{R}}\right.$; Fig. 9) showed a good correlation, with no bias and an average absolute difference of $0.06 \%$ $\pm 0.17 \%$.

TABLE 2. Summary of spring opening and force values at the different time points

\begin{tabular}{lccccc}
\hline \multirow{2}{*}{$\begin{array}{l}\text { Time } \\
\text { Point }\end{array}$} & \multicolumn{2}{c}{ Combined Opening $(\mathrm{mm})$} & & \multicolumn{2}{c}{ Combined Force $(\mathrm{N})$} \\
\cline { 2 - 3 } \cline { 5 - 6 } & Mean \pm SD & $\mathrm{p}$ Value & & Mean \pm SD & $\mathrm{p}$ Value \\
\hline Insertion & $30.1 \pm 4.3$ & 0.002 & & $23.7 \pm 8.6$ & $<0.001$ \\
\hline FU1 & $43.7 \pm 6.0$ & 0.03 & & $10.3 \pm 4.7$ & 0.001 \\
\hline FU2 & $53.1 \pm 3.8$ & 0.01 & & $2.5 \pm 3.3$ & $\mathrm{NS}$ \\
\hline Removal & $55.0 \pm 2.6$ & $\mathrm{NS}$ & & $1.6 \pm 2.4$ & $\mathrm{NS}$ \\
\hline
\end{tabular}

NS = not significant.

The $p$ values indicate significant correlation with age.

\section{Discussion}

Spring-assisted cranioplasty is an appealing method for treating sagittal synostosis-related scaphocephaly, with some potential advantages (less surgical time, fewer blood transfusions, shorter hospital stay) over the "traditional" formal cranial vault remodeling surgery. However, current wire form springs for sagittal cranioplasty are often shaped in the surgical theater, thus lacking uniformity and reproducibility. Studies in the literature to date have focused on estimating spring distraction force in patients
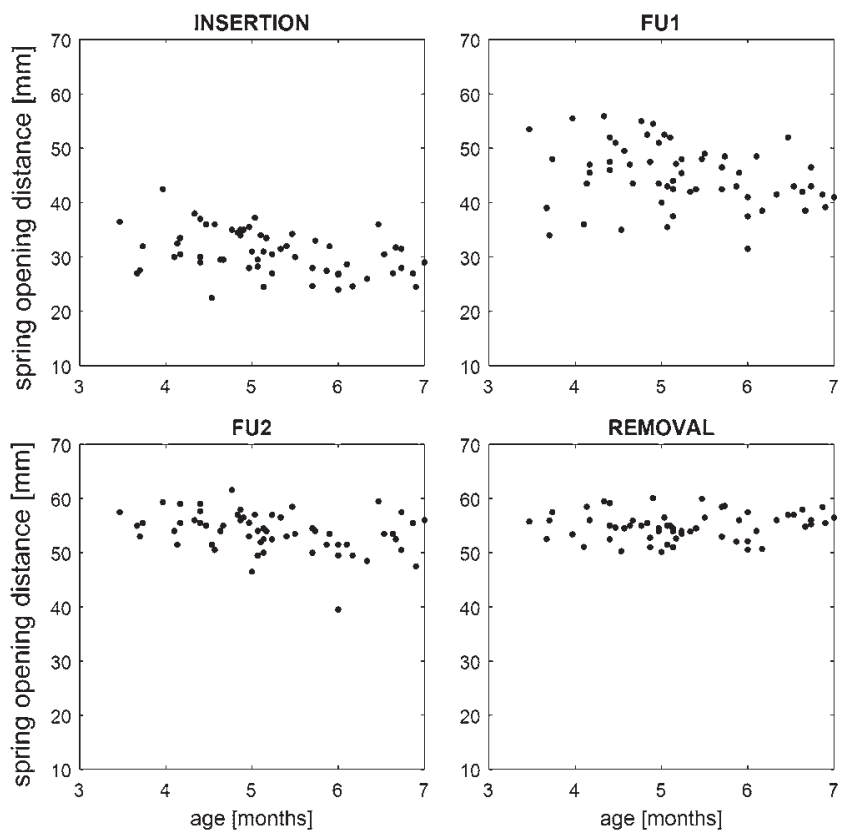

FIG. 6. Distribution of combined spring opening distance values by patient age, at the different time points. 

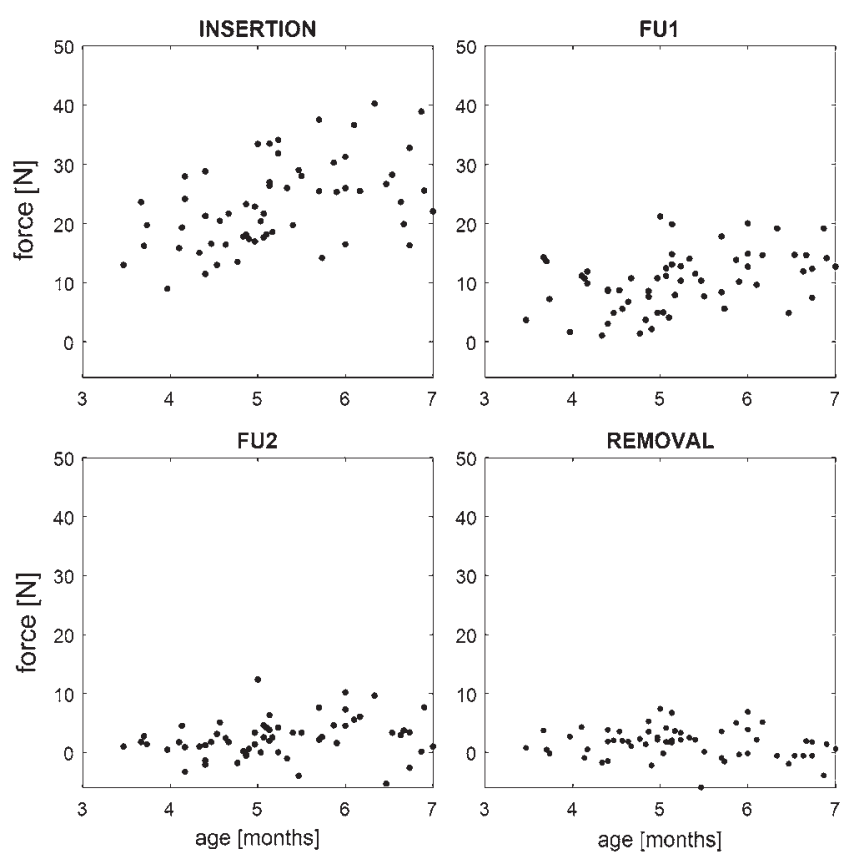

FIG. 7. Distribution of combined spring force values by patient age, at the different time points.

at the time of implantation, with no information about its dissipation in the long term. In the current study, an engineering approach was applied to assess the performance of springs over time in a population of children with sagittal synostosis treated with a preformed set of devices and a standardized surgical approach. A numerical model for estimating spring force from follow-up in vivo images was derived and used to assess spring-skull interaction from the time of spring insertion to removal. A population analysis was performed to understand the differences in mechanical behavior and results between spring models, their combined effect on each patient skull, and the relationship with patient age in terms of final clinical outcome.

Results from the present study are similar to those reported from other centers. The duration of spring treatment in our series $(110.9 \pm 53.6$ days, range $28-300$ days) was in the same time frame as that described elsewhere. ${ }^{2,12,15,17,19}$ The force at insertion in our population $(11.4 \pm 4.3 \mathrm{~N}$ for anterior spring, $11.8 \pm 4.1 \mathrm{~N}$ for posterior spring, resulting in a total force of $23.2 \pm 7.2 \mathrm{~N}$ applied to the skull) was in the upper end of the range of forces per device reported in other studies (range 5-12 N). ${ }^{2,11-13,15,17,19}$ Spring stiffness is not stated in most studies; however, considering the initial opening and force recorded from data in the literature, the GOSH springs are, on average, stiffer than those used at other centers. Analysis of the spring mechanical behavior at different crimping distances showed that standardization of the implantation procedure is key to achieve predictable spring forces, as is on-table expansion and final spring opening distance. The fact that the distance to which the spring is crimped during insertion affects the force exerted by the spring and the degree to which it will open presents a significant problem in ensuring a precise end point. The problem arises from the mechanical prop-

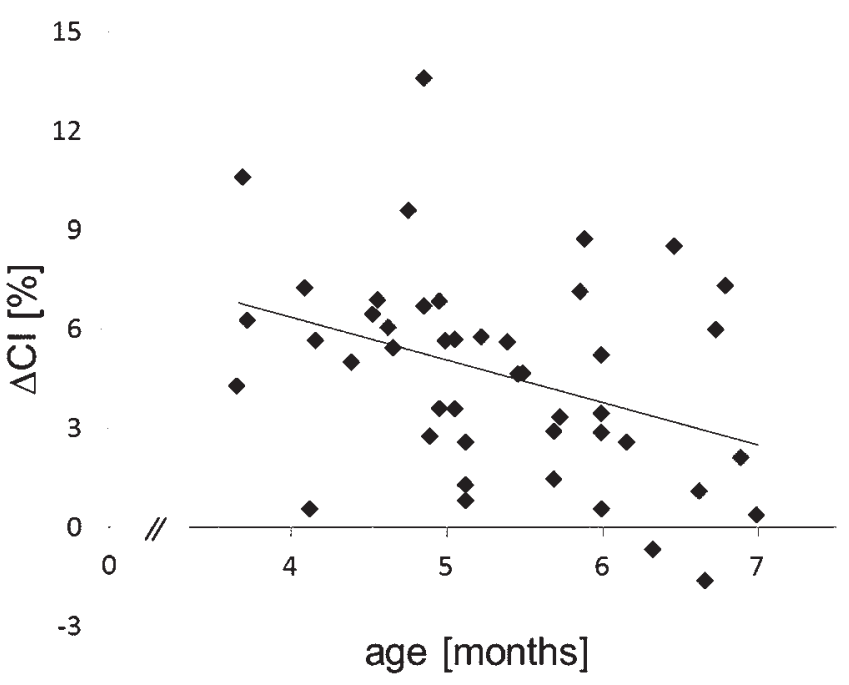

FIG. 8. Correlation between patient age and the change in cephalic index $(\Delta \mathrm{Cl})$.

erties of steel. Each time a steel spring is activated, there is a small amount of plastic deformation, which means that the spring will not fully recover when the forces are dissipated. Although precise reproducible manufacturing and insertion techniques can reduce the degree of plastic deformation, they cannot eradicate the problem, and future development should focus on different biocompatible materials

Our data indicated a surgeon preference for stiffer individual springs in older patients, resulting in a stiffer combination of springs acting on each older patient skull as compared with the other spring models. This means that when analyzing performance of the springs on the operating table, there was no difference in the openings between the different models as this is driven by the surgeon's choice to implant distractors that provide an initial opening of approximately $3 \mathrm{~cm}$. However, when looking at the force distribution on the operating table, there are differences in the initial force between models since, despite the similar openings, stiffer springs provide higher forces both individually and when analyzed in combination as a system in the patient. To achieve the same initial opening, stiffer springs have to be used in older children.

It is interesting to note that with time the differences in force applied to the skull by the different models disappeared since all spring models achieved similar openings with similar residual forces at removal. The choice of spring model influences the initial behavior of the device but not the final result. Furthermore, our spring kinematics analysis showed that every spring combination in the patient population had virtually stopped expanding after 10 days of insertion, meaning that current devices stop exerting expansion force long before their removal. The inherent viscoelasticity of the pediatric skull accommodates forces and with time allows full expansion regardless of the model used. This is in line with the results in a population of rabbits described by Davis et al., in which $80 \%$ of the expansion was achieved within 2 weeks. In addition, older patients require larger spring forces and smaller 


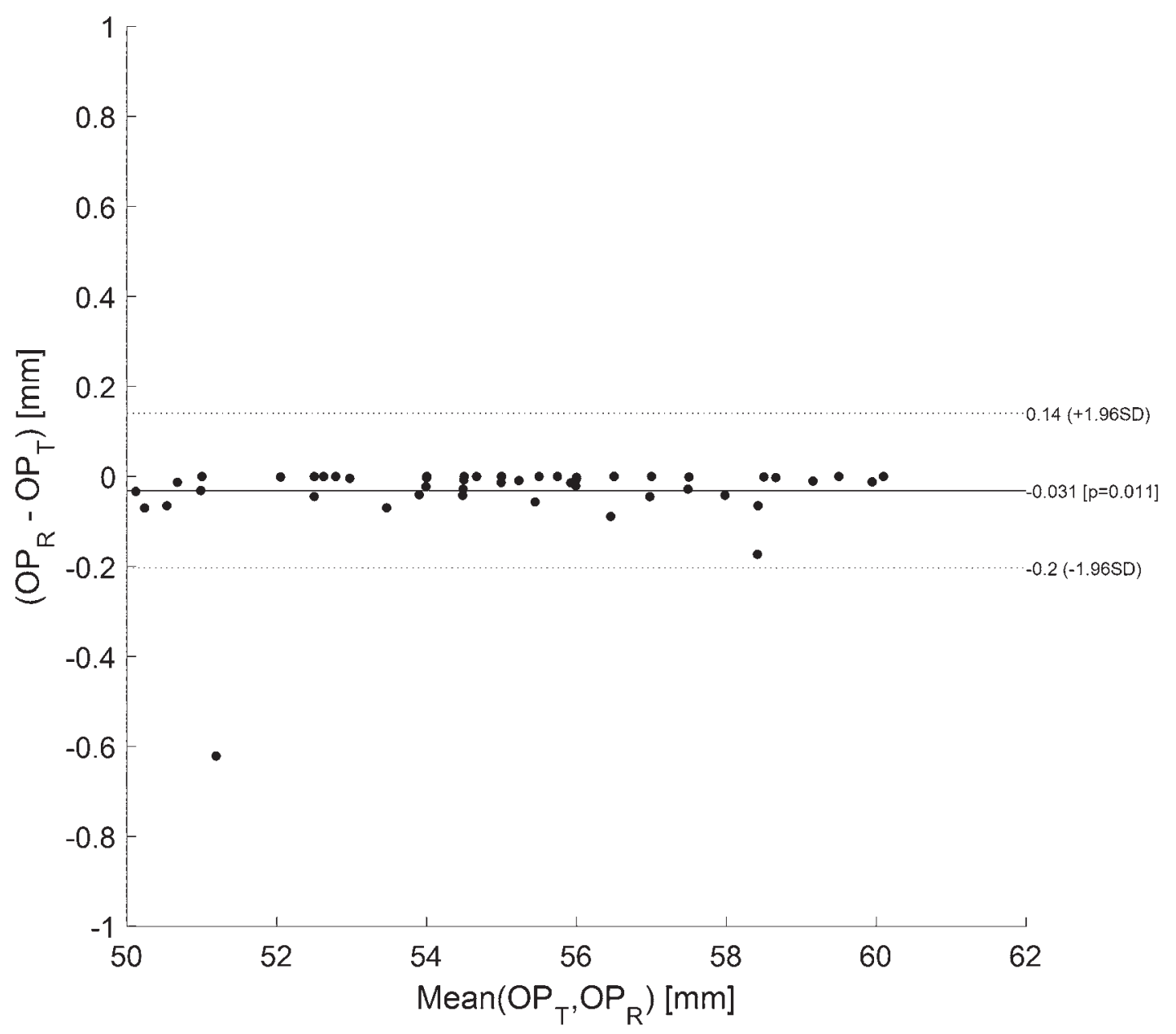

FIG. 9. Bland-Altman plot of the comparison between the spring opening at removal $\left(O P_{R}\right)$ and the opening at 10 days $\left(O P_{T}\right)$.

openings on the operating table as well as at the initial follow-up compared with those in younger children, but all the springs approximate a plateau after 10 days with no evident opening and force differences in older versus younger cases. These results raise the question about the ideal force required. The clinically selected forces are adequate, but given the fact that all springs reached the same end point, it is possible that the same results could have been achieved in older children by using springs exerting a smaller force. As the effectiveness of a spring lies in its ability to exert forces on the patient skull, understanding the forces involved in the distraction process will drive the design of new distractors. Utilizing this observation, our unit is currently developing a modification of our distraction device that aims to dissipate the force in a more gradual fashion.

In the present study, the performance of cranioplasty springs was assessed in terms of spring opening distance. In our cohort, springs opened to a mean of $55.0 \pm 2.6$ $\mathrm{mm}$; hence, we believe that all springs achieved satisfactory distraction. Overall assessment of successful cranioplasty would require an analysis of satisfactory aesthetic outcomes, which depend not only on the spring behavior but also on other parameters and external factors such as the initial severity of the craniosynostosis and the overall skull remodeling. ${ }^{16}$ While every case of scaphocephaly is individual in its aesthetic parameters and bone morphology, by undertaking a uniform standardized approach in all of these cases, we hereby present a core volume of data on which to build up and further this minimal access technique by creating patient-specific distractors and osteotomies for bespoke outcomes. This is ongoing work within our department.

When looking at changes in the cephalic index as a parameter to assess results of the operation in the geometric correction of scaphocephaly, we noticed that the skulls of the older patients in our population at the 3-week followup seemed to have reshaped less than those in the younger children; that is, the spring may be less effective in the treatment of scaphocephaly in older patients, as also reported by other authors. ${ }^{15}$ Our analysis showed that the final spring opening distance was not dependent on age; perhaps, despite similar interparietal widening, the anteroposterior shortening is less pronounced in older patients since the skull system is stiffer at this age, with a weaker mechanical coupling of the two directions. Further studies will be required to assess the long-term effect of the remodeling, as the process could just be slower in older chil- 
dren, effectively achieving the same skull shape changes with time. The cephalic index is an imperfect measurement for assessing the degree of scaphocephaly correction as it focuses on only one aspect of the deformity and does not take into account other features, such as frontal bossing, bitemporal "pinching," low posterior vertex, and occipital protuberance. On-table 3D imaging proved to be a suitable means of assessing the long-term effects of spring cranioplasty, as demonstrated in a recent publication by our group..$^{18}$

In this retrospective study, we aimed to provide information on spring cranioplasty at GOSH between 2010 and 2013. Current practice in selecting the spring model is to test progressively stiffer springs until an on-table opening of at least $3 \mathrm{~cm}$ is achieved and to record the final spring model. Although it may be possible to prospectively assess the long-term outcome of springs in patients who had on-table suboptimal spring opening in order to determine whether the final outcome is independent of distractor selection (as it appears from these results), our group's efforts are currently directed toward prospectively predicting the outcome of spring cranioplasty by means of numerical modeling of spring expansion in accurate patient-specific models. ${ }^{1}$ This is a key benefit of standardization of the devices and the technique and allows us to improve surgical planning and the process of informed consent in an individualized, patient-specific manner.

Finally, 50\% of the GOSH spring recovery happens on the operating table: such an early, sudden expansion of the skull may be undesirable and may be connected with device dislodgement, skull erosion, difficulty of spring removal, and other complications reported in spring-assisted cranioplasty. ${ }^{2,5,12,19}$ Current spring distractors, although they are performing according to the planned design, may be too simple to achieve satisfactory results, presenting complications in the short term and suboptimal reshaping of the skull as a final outcome, especially in older patients. Future studies should address a more complex device design that allows for larger potential expansions and more control of the opening and force exertion during the implantation period.

\section{Conclusions}

The GOSH springs employed in the treatment of sagittal craniosynostosis work in a predictable and reliable manner: patient age as well as the spring model has an initial transitory effect on the spring opening distance and force exerted to the patient skull, but long-term results show that most of the expansion is achieved within 10 days from insertion in all cases. Using precisely manufactured spring distractors and avoiding excessive crimping during insertion improve the predictability of spring opening distance. The choice of distractor is dictated by on-table performance, but the pediatric skull accommodates the spring force regardless of the initial conditions (spring model and patient age). Further understanding of spring and skull biomechanics will inspire and guide advances in this important surgical technique for the correction of scaphocephaly.

\section{Acknowledgments}

This work has been funded by the Great Ormond Street Hospital for Children Charity (Grant No. 12SG15). We thank The Active Spring Company UK for their technical assistance.

This report incorporates independent research from the National Institute for Health Research Biomedical Research Centre funding scheme. The views expressed in this publication are those of the author(s) and not necessarily those of the National Health Service, National Institute for Health Research, or Department of Health.

\section{References}

1. Borghi A, Rodriguez-Florez N, Dunaway DJ, Jeelani NU, Schievano S: Biomechanics of spring cranioplasty: 3D ontable scanning to validate a model of cranial distraction, in Proceedings of 22nd Congress of the European Society of Biomechanics, Lyon, France, 2016 (https://esbiomech. org/conference/index.php/congress/lyon2016/paper/ viewFile/827/12) [Accessed July 5, 2017]

2. David LR, Plikaitis CM, Couture D, Glazier SS, Argenta LC: Outcome analysis of our first 75 spring-assisted surgeries for scaphocephaly. J Craniofac Surg 21:3-9, 2010

3. Davis C, Windh P, Lauritzen CGK: Adaptation of the cranium to spring cranioplasty forces. Childs Nerv Syst 26:367371,2010

4. Davis C, Windh P, Lauritzen CGK: Cranial bone and suture strains incident to spring-assisted cranioplasty. Plast Reconstr Surg 125:1104-1110, 2010

5. Davis C, Windh P, Lauritzen CGK: Do expansile cranial springs erode through the cranium? J Craniofac Surg 20:168-170, 2009

6. Davis C, Windh P, Lauritzen CGK: Spring-assisted cranioplasty alters the growth vectors of adjacent cranial sutures. Plast Reconstr Surg 123:470-474, 2009

7. Davis C, Windh P, Lauritzen CGK: Spring expansion is influenced by cranial biomechanics. J Craniofac Surg 21:843846, 2010

8. de Faria Valle Dornelles R, Cardim VLN, de Campos Fonseca Pinto ACB, Alonso N: Skull base cephalometric changes in cranial expansion by springs. J Craniofac Surg 22:1496-1501, 2011

9. Guimarães-Ferreira J, Gewalli F, David L, Olsson R, Friede H, Lauritzen CGK: Spring-mediated cranioplasty compared with the modified pi-plasty for sagittal synostosis. Scand J Plast Reconstr Surg Hand Surg 37:208-215, 2003

10. Haliasos N, Nazir M, DeSouza R, Gwanmesia I, Jeelani O: Mathematical model for the standardization of radiologically measured spring apertures in children with craniosynostosis, in International Society for Craniofacial Surgery 14th Biennial International Congress. Livingston, Zambia: ISCFS, 2011

11. Lauritzen C, Sugawara Y, Kocabalkan O, Olsson R: Spring mediated dynamic craniofacial reshaping. Case report. Scand J Plast Reconstr Surg Hand Surg 32:331-338, 1998

12. Lauritzen CGK, Davis C, Ivarsson A, Sanger C, Hewitt TD: The evolving role of springs in craniofacial surgery: the first 100 clinical cases. Plast Reconstr Surg 121:545-554, 2008

13. Mackenzie KA, Davis C, Yang A, MacFarlane MR: Evolution of surgery for sagittal synostosis: the role of new technologies. J Craniofac Surg 20:129-133, 2009

14. Prange MT, Luck JF, Dibb A, Van Ee CA, Nightingale RW, Myers BS: Mechanical properties and anthropometry of the human infant head. Stapp Car Crash J 48:279-299, 2004

15. Pyle J, Glazier S, Couture D, Sanger C, Gordon S, David L: Spring-assisted surgery-a surgeon's manual for the manufacture and utilization of springs in craniofacial surgery. $\mathbf{J}$ Craniofac Surg 20:1962-1968, 2009 
16. Rodgers W, Glass GE, Schievano S, Borghi A, RodriguezFlorez N, Tahim A, et al: Spring-assisted cranioplasty for the correction of nonsyndromic scaphocephaly: a quantitative analysis of 100 consecutive cases. Plast Reconstr Surg 140:125-134, 2017

17. Taylor JA, Maugans TA: Comparison of spring-mediated cranioplasty to minimally invasive strip craniectomy and barrel staving for early treatment of sagittal craniosynostosis. J Craniofac Surg 22:1225-1229, 2011

18. Tenhagen M, Bruse JL, Rodriguez-Florez N, Angullia F, Borghi A, Koudstaal MJ, et al: Three-dimensional handheld scanning to quantify head-shape changes in spring-assisted surgery for sagittal craniosynostosis. J Craniofac Surg 27:2117-2123, 2016

19. van Veelen MLC, Mathijssen IMJ: Spring-assisted correction of sagittal suture synostosis. Childs Nerv Syst 28:1347-1351, 2012

\section{Disclosures}

The authors report no conflict of interest concerning the materials or methods used in this study or the findings specified in this paper.

\section{Author Contributions}

Conception and design: Borghi. Acquisition of data: Borghi, McNicholas. Analysis and interpretation of data: Borghi, Schievano. Drafting the article: Borghi, Schievano. Critically revising the article: Borghi, Schievano, Rodriguez Florez, Rodgers, Ponniah, James, Hayward, Dunaway, Jeelani. Reviewed submitted version of manuscript: Borghi, Schievano, Rodriguez Florez, Rodgers, James, Hayward. Approved the final version of the manuscript on behalf of all authors: Borghi. Statistical analysis: Borghi, Rodriguez Florez. Administrative/technical/material support: Schievano. Study supervision: Borghi, Schievano, Dunaway, Jeelani.

\section{Supplemental Information}

\section{Previous Presentations}

Parts of these results were presented in abstract form at the annual meetings of the International Society of Craniofacial Surgery held in Tokyo, Japan, in September 2015, and the International Society for Paediatric Neurosurgery held in Izmir, Turkey, in October 2015.

\section{Correspondence}

Alessandro Borghi, UCL Institute of Child Health, 30 Guilford St., London WC1N 1EH, United Kingdom. email: a.borghi@ucl. ac.uk. 